\title{
Quantum statistics and Altarelli-Parisi evolution equations
}

\author{
G. Mangano, G. Miele and G. Migliore \\ Dipartimento di Scienze Fisiche, Università di Napoli - Federico II -, and INFN - Sezione di \\ Napoli, Mostra D'Oltremare Pad. 20, 80125, Napoli, Italy.
}

\begin{abstract}
The phenomenological evidence of quantum statistical effects in parton physics is here briefly summarized, and the recent good results obtained by parameterizing the parton distributions in terms of Fermi-Dirac and Bose-Einstein statistical functions are discussed. In this framework we study the modification of the scaling behaviour of parton distributions due to quantum statistical effects. In particular, by following a well-known formal analogy which holds between the Altarelli-Parisi evolution equations, at leading-log approximation, and a set of Boltzmann equations, we suggest a generalization of evolution equations to take into account Pauli exclusion principle and gluon induced emission.
\end{abstract}

PACS number: 13.60.-r

published in Nuovo Cim. A108 (1995) 867-882. 


\section{Introduction}

The low $x$ regime in deep inelastic processes has recently received much attention due, in particular, to the advent of the HERA electron-proton machine, which would provide precision measurements in the region $Q^{2}>10 \mathrm{GeV}^{2}$ and $x \geq 10^{-4}$ [1]. In the limit of very small momentum fraction one deals with a dense system of partons in a weak coupling limit, in which, however, interactions among partons cannot be neglected, being able to build up, as we know from many cases in condensed matter physics, a collective dynamics. The aim of this paper is to study the effect of statistical correlations among partons, due to their Fermi or Bose nature, in the $Q^{2}$ evolution of their distribution functions. These correlations, in fact, would be expected whenever the parton wave functions overlap.

In terms of the two phase-space variables $Q^{2}$ and $x$, it is possible to distinguish three regions in which strong interactions among partons, dictated by QCD, behave quite differently:

1) For high values of $Q^{2}$ and small densities $\rho$, defined as the number $N$ of partons per unit of rapidity $y=\log (1 / x)$ in the transverse plane

$$
\rho=\frac{d N}{d y} \frac{1}{\pi R_{h}^{2}}
$$

with $R_{h}$ the radius of the hadron, one can powerfully apply the perturbative QCD methods. In particular, the $Q^{2}$ evolution of structure functions can be evaluated at leading-log level in $Q^{2}$ by standard Altarelli-Parisi equations (AP) [2] when the following conditions are satisfied: $\alpha_{s}<<1, \alpha_{s} \log Q^{2} \approx 1$, and $\alpha_{s} \log (1 / x)<<1$. Alternatively, in the kinematical region: $\alpha_{s} \log Q^{2}<<1$, and $\alpha_{s} \log (1 / x) \approx 1$ and still with $\alpha_{s}<<1$, the approach of extracting the contribution of the order $\left[\alpha_{s} \log (1 / x)\right]^{n}$, leads to Fadin-Kuraev-Lipatov equations (FKL) [3]

2) The low $Q^{2}$ regime, or equivalently the long-distance interaction region, is typically the realm of non-perturbative QCD. In this case, the value of the strong coupling constant is large and one is dealing with the confinement problem.

3) Finally, for high $Q^{2}$ and large densities, as already mentioned, we are still in nonperturbative conditions, but in this case, the latter are rather due to the large number of partons which interact each other. This high density QCD regime is particularly interesting from a theoretical point of view, since the small value of the coupling constant 
gives us a chance to successfully face the problem. Many attempts have been already addressed to understand the main characteristics of this parton-plasma dynamics. In particular, due to interactions among partons, one should expect nonlinear effects in the $Q^{2}$ evolution of distribution functions: stated differently, microscopical processes with two or more partons in the initial state become relevant in changing their resulting number. This point of view is at the basis of Gribov-Levin-Ryskin equations (GLR) [4, where besides parton decays, whose probability is proportional to $\alpha_{s} \rho$, parton annihilation processes are explicitly taken into account. These introduce in the scaling equations a quadratic term in the distribution functions of the form $\alpha_{s}^{2} \rho^{2} / Q^{2}$. Hence, one gets for the particle balance in a cell of the phase-space a Vlasov equation

$$
\frac{\partial^{2} \rho}{\partial y \partial \log Q^{2}}=\frac{3 \alpha_{s}}{\pi} \rho-\frac{\alpha_{s}^{2}}{Q^{2}} \rho^{2} \text {. const. . }
$$

One comment is in order at this point: the nonlinear effects expected in the evolution can be both of dynamical and/or statistical nature. The first ones are simply due to interaction processes among partons which are very close each other, and are the ones included to some extent in Eq. (2). However, in a dense medium quantum statistics may provide similar relevant effects, related in particular to exchange interactions for Fermi particles (quarks) and induced emission processes for Bosons (gluons). Despite of this in the literature this point has not been sufficiently remarked. The decay process, for example, of a parton into a quark with a definite momentum, if it occurs in presence of many other quarks with the same momentum, would be strongly suppressed. In this case, the corresponding probability would be not only proportional to the decaying parton distribution function, but also to a Pauli blocking factor, which depends on the final quark density.

In order to find a simple way in which the statistical effects can be taken into account in the evolution equations, we will start from a phenomenological description of parton distributions in terms of equilibrium-like functions [5]. This thermodynamical approach to deep inelastic scattering phenomena was developed on the basis of previous papers [6], where Gottfried sum rule violation [7], [8] and other typical behaviour of structure functions are interpreted in terms of Pauli exclusion principle. In section 2 we will briefly review this idea, showing the good agreement of the theoretical predictions for unpolarized and polarized structure functions with NMC, EMC and E142 experimental data [8], [9], [10]. This agreement is not completely surprising if one stresses the fact that, as shown in [11], the $Q^{2}$-evolution can be consistently viewed as a thermalization process. According to this analysis, the AP scaling equations at leading-log can be 
shown to be equivalent to a set of Boltzmann transport equations, which, as wellknown, describe the approach towards equilibrium conditions of a thermodynamical system, where a simple function of $Q^{2}$ plays the role of time. In this sense one can guess, in strict analogy with H-Boltzmann theorem, that parton distributions would asymptotically reach equilibrium shape at infinite $Q^{2}$. The good agreement with the data of equilibrium-like distributions at $Q^{2}=4 \mathrm{GeV}^{2}$, could therefore suggest that thermalization process is quite rapid.

The stated analogy between transport equations and AP straightforwardly leads to a generalization of the latter in a regime of quite large densities [12]. In fact, AP are strictly equivalent only to a set of Boltzmann equations for a very dilute system, where all quantum statistical effects, namely Pauli blocking and induced gluon emission, are negligible. These can be simply introduced by adding in the collisional integral appropriate factors of the form $(1 \pm f)$, where $f$ are parton statistical functions, namely their distributions once the level degeneracy has been subtracted out (see section 3). This procedure leads to a set of generalized nonlinear AP equations, which recover the usual AP in the low density region, but whose validity is quite wider, since the effects originated by quantum statistics have been explicitly taken into account.

The paper is organized as follow: in section 2 we briefly review main experimental results leading to the conclusion that quantum statistics may play a role in parton dynamics inside hadrons. The thermodynamical model proposed in [5] is also described. In section 3 we will show, following [11], that AP equations can be formally viewed as transport equations: this can be achieved by considering non regularized splitting functions and by explicitly computing infrared virtual gluon contributions. The generalization of AP to a new set of nonlinear equations which contain quantum statistics effects is the subject of section 4. Finally in section 5 we give our conclusions and remarks.

\section{Pauli exclusion principle in deep inelastic scat- tering}

\subsection{Experimental results}

Deep inelastic experiments seem to be an inexhaustible source of information on the hadronic structure and continue to considerably improve our understanding of strong interaction dynamics. A measurement of proton and neutron $F_{2}(x)$ structure function

performed by the NMC Collaboration at CERN [8] suggests a rather large $S U(2)$ flavour 
breaking in the sea quark [13]. In particular they have obtained a determination for the difference

$$
\mathcal{I}_{G}=\int_{0}^{1} \frac{d y}{y}\left[F_{2}^{p}(y)-F_{2}^{n}(y)\right]=0.235 \pm 0.026
$$

instead of the value $1 / 3$ predicted by an $S U(2)$ symmetric sea; in fact

$$
\mathcal{I}_{G}=\frac{1}{3}(u+\bar{u}-d-\bar{d})=\frac{1}{3}+\frac{2}{3}(\bar{u}-\bar{d})
$$

This result, which represents a relevant violation of the Gottfried sum rule [7], yields

$$
\bar{d}-\bar{u}=\int_{0}^{1} d x[\bar{d}(x)-\bar{u}(x)]=0.15 \pm .04 \quad .
$$

The inequality $\bar{d}>\bar{u}$, however, was already argued many years ago by Field and Feynman [14 on pure statistical basis. They suggested that in the proton the production from gluon decays of $u \bar{u}$-pairs with respect to $d \bar{d}$-pairs would be suppressed by Pauli principle because of the presence of two valence $u$ quarks but of only one valence $d$ quark. Assuming this point of view, the experimental result (3) naturally leads to the conclusion that quantum statistical effects would play a sensible role in parton dynamics and that, in particular, parton distribution functions are affected by them. In this picture one may also easily account for the known dominance at high $x$ of $u$-quarks over $d$-quarks, whose characteristic signature is the fast decreasing of the ratio $F_{2}^{n}(x) / F_{2}^{p}(x)$ in this regime. Fermi statistics imply, in fact, a broader distribution for $u$ quarks, due to their larger abundance.

Another evidence for the effect of the Pauli principle on the parton structure follows from the double helicity asymmetry for polarized muon (electron) - polarized proton deep inelastic scattering $A_{1}^{p}(x)$. By denoting with $q^{+}(x)\left(q^{-}(x)\right)$ quark distributions with helicity parallel (antiparallel) to the proton helicity, $A_{1}^{p}(x)$ is defined as

$$
A_{1}^{p}(x) \equiv \frac{g_{1}^{p}(x)}{F_{1}^{p}(x)} \approx \frac{4\left[u^{+}(x)-u^{-}(x)\right]+\left[d^{+}(x)-d^{-}(x)\right]}{4 u(x)+d(x)} .
$$

Experimentally this quantity increases towards unity for high $x$ [9], thus in this regime $u^{+}(x)$ dominates over $u^{-}(x), d^{+}(x)$, and $d^{-}(x)$. This interesting behaviour can be interpreted reminding that at $Q^{2}=0$ the first momenta of the valence quark distributions are related to the axial couplings $F$ and $D$ through the following relations

$$
u_{v a l}^{+}=1+F, \quad u_{v a l}^{-}=1-F, \quad d_{\text {val }}^{+}=\frac{1+F-D}{2}, \quad d_{\text {val }}^{-}=\frac{1-F+D}{2} .
$$


Reminding that $F=0.477 \pm .011 \approx 1 / 2$ and $D=.755 \pm .011 \approx 3 / 4$ [15], we get for the valence quark abundances $u_{\text {val }}^{+} \approx 3 / 2, u_{\text {val }}^{-} \approx 1 / 2, d_{\text {val }}^{+} \approx 3 / 8$ and $d_{\text {val }}^{-} \approx$ $5 / 8$. The fact that dominant distributions correspond to highest values of the valence abundances gives the abundance - shape correlation, which is the typical property of the Fermi - Dirac distribution function: larger abundances correspond to broader distributions. In particular, from the previously obtained values for the first momenta one can extrapolate the useful relation valid for the quark distributions [6]

$$
u^{-}(x)=\frac{1}{2} d(x)
$$

which leads to

$$
\Delta u(x) \equiv u^{+}(x)-u^{-}(x)=u(x)-d(x) \quad .
$$

This equation allows to relate the contribution in the proton polarized structure function $g_{1}^{p}(x)$ due to the $u$ quarks to the one due to $u$ and $d$ present in $F_{2}^{p}(x)-F_{2}^{n}(x)$, i.e.

$$
\left.\left.x g_{1}^{p}(x)\right|_{u} \approx \frac{2}{3}\left(F_{2}^{p}(x)-F_{2}^{n}(x)\right)\right|_{u+d}
$$

Then, neglecting the $d$ quarks term in $g_{1}^{p}(x)\left(\Delta d_{v a l}=-1 / 4 \Delta u_{v a l}\right.$ and $\left.e_{d}^{2}=1 / 4 e_{u}^{2}\right)$, we get

$$
x g_{1}^{p}(x) \approx \frac{2}{3}\left(F_{2}^{p}(x)-F_{2}^{n}(x)\right)
$$

at least in the region dominated by valence quarks. This relation is in good agreement with the experiment [8], [9].

\subsection{Quantum statistical approach to parton distributions}

In a recent paper [5], the idea to extensively consider Pauli principle and use FermiDirac and Bose-Einstein statistics for the parton distributions has been developed. It has succeeded in making reasonable assumptions for various polarized parton distributions in terms of unpolarized ones, explaining the observed violation of Ellis-Jaffe sum rule [16], and giving a possible solution to the spin crisis problem [9].

In this framework the quark distributions are parameterized in terms of Fermi-Dirac statistical functions as

$$
q_{a}(x)=f(x)\left[\exp \left(\frac{x-\tilde{x}\left(q_{a}\right)}{\bar{x}}\right)+1\right]^{-1} .
$$


Here $\tilde{x}\left(q_{a}\right)$ plays the role of the thermodynamical potential, $\bar{x}$ of the temperature and $f(x)$ is the level-density in the $x$ variable. This function is ultimately related to the non perturbative dynamics responsible for the binding of quarks and gluons inside the hadrons, so it is theoretically undetermined. Analogously for the gluons (we neglect their polarization) the Bose-Einstein relation has been assumed

$$
G(x)=\frac{16}{3} f(x)\left[\exp \left(\frac{x-\tilde{x}(G)}{\bar{x}}\right)-1\right]^{-1},
$$

where now the factor $16 / 3$ is due to the colour degeneracy with respect to the quarks case and to the sum over the two helicity states. Notice that the weight function $f(x)$ has been assumed universal, being the same in (12) and (13). Moreover, the previous considerations allow also to assume the relation

$$
d(x)=\frac{u^{-}(x)}{1-F}
$$

and a dipole approximation for the $d$-quark polarization, namely

$$
\Delta d(x)=-k f(x) \exp \left(\frac{x-\tilde{x}\left(u^{-}\right)}{\bar{x}}\right)\left[\exp \left(\frac{x-\tilde{x}\left(u^{-}\right)}{\bar{x}}\right)+1\right]^{-2} .
$$

In terms of this parameterization it is possible to reproduce the NMC data [8] for $F_{2}^{p}(x)$ and $F_{2}^{n}(x)$ taken at $Q^{2}=4 G e V^{2}$. These predictions are also compatible with the antiquark data obtained from neutrino deep inelastic scattering [17, and the results known for the gluon distribution [18], [19]. In particular, from the fit procedure it has been obtained $A=0.579, \alpha=-0.845, k=0.769, \bar{x}=0.132, \tilde{x}\left(u^{+}\right)=0.524, \tilde{x}\left(u^{-}\right)=$ $0.143, \tilde{x}\left(\bar{u}^{+}\right)=-0.216, \tilde{x}\left(\bar{d}^{+}\right)=\tilde{x}\left(\bar{d}^{-}\right)=\tilde{x}\left(\bar{u}^{-}\right)=-0.141$, and $\tilde{x}(G)=-0.012$ [5, where for the function $f(x)$ the following form was assumed

$$
f(x)=A x^{\alpha},
$$

to match the singular behaviour of parton distribution at low $x$. In Figures 1 and 2 we show the good agreement between the theoretical predictions of this model and the experimental data for $F_{2}^{p}(x)-F_{2}^{n}(x)$ and $F_{2}^{n}(x) / F_{2}^{p}(x)$, whereas, in Figures 3 and 4 we report the predictions for the polarized structure functions $x g_{1}^{p}(x)$ and $x g_{1}^{n}(x)$, which fits quite well with the experimental data [9], [10].

The analysis performed so far is at fixed $Q^{2}=4 \mathrm{GeV}^{2}$. In order to consider also the experimental data at different $Q^{2}$ a scaling evolution equation is needed. In the large $x$ 
and $Q^{2}$ regime, AP equations provide a reliable description for scaling [2]. As already mentioned in the Introduction, the low $x$ region is characterized by an overdense parton medium, so one has to expect nonlinear effects in the evolution equations due to the overlapping of parton wave-functions. Thus, a quite natural conclusion is that a set of generalized AP equations which would describe the evolution in the moderately low $x$ region, should take into account quantum statistical effects. A way to approach this problem is to start from the analogy showed in [11] occurring between standard leading-log AP equations and Boltzmann transport equations.

\section{Altarelli-Parisi evolution equations as a set of Boltzmann equations}

As well-known, the logarithmic dependence on $Q^{2}$ of the parton distribution momenta, predicted in the framework of perturbative $Q C D$, has a simple and beautiful interpretation in terms of evolution equations for parton distribution functions [2]. At leading-log level, the AP equations can be written in the following way

$$
\frac{d}{d t} p_{A}(x, t)=\frac{\alpha_{s}(t)}{2 \pi} \int_{x}^{1} \frac{d y}{y} \sum_{B} p_{B}(y, t) P_{A B}\left(\frac{x}{y}\right),
$$

where $t=\ln \left(Q^{2} / \mu^{2}\right), \mu$ is some renormalization scale and $p_{A}(x, t)$ denote the parton distribution functions ( $A, B=$ quarks, antiquarks and gluons). By defining

$$
\tau \equiv \frac{1}{2 \pi b} \ln \left[\frac{\alpha_{s}(0)}{\alpha_{s}(t)}\right],
$$

with $b \equiv\left(33-2 n_{f}\right) /(12 \pi)\left(n_{f}\right.$ is the number of flavours), Eq. (17) becomes

$$
\frac{d}{d \tau} p_{A}(x, \tau)=\int_{x}^{1} \frac{d y}{y} \sum_{B} p_{B}(y, \tau) P_{A B}\left(\frac{x}{y}\right) \text {. }
$$

Note that the dependence on $\tau$ of r.h.s. of (19) comes only through $p_{B}(y, \tau)$. In Eqs. (17) and (19), $P_{A B}(x / y)$ stand for the splitting functions, evaluated by using standard equivalent parton method. They correspond to the probability for the elementary threebody processes to occur in which a parton with momentum fraction $x$ is produced by a parton with higher fraction $y=x / z$. Following the original Altarelli-Parisi approach, the $1 /(1-z)$ singularities of $P_{A A}$, are removed by introducing the $(1-z)+$ regularization prescription凹, which explicitly implements the cancellation occurring between the real

\footnotetext{
${ }^{1}$ The integrals are defined in this prescription by $\int_{0}^{1}\left[f(z) /(1-z)_{+}\right] d z \equiv \int_{0}^{1}[f(z)-f(1)] /(1-z) d z$
} 
and virtual soft gluons emissions. In this way one gets

$$
\begin{aligned}
P_{q q} & =\frac{4}{3}\left[\frac{1+z^{2}}{(1-z)_{+}}+\frac{3}{2} \delta(1-z)\right], \\
P_{q g} & =\frac{1}{2}\left[z^{2}+(1-z)^{2}\right], \\
P_{g q} & =\frac{4}{3} \frac{1+(1-z)^{2}}{z}, \\
P_{g g} & =6\left[z(1-z)+\frac{1-z}{z}+\frac{z}{(1-z)_{+}}\right]+2 \pi b \delta(1-z) .
\end{aligned}
$$

The microscopic picture beyond the scaling violation equations (19), however, has one main difficulty: the splitting functions (20)-(23) cannot be all interpreted, strictly speaking, as probability densities, since they are not positive definite (e.g. $\int_{0}^{1} P_{q q}(z) d z=$ 0 ). This, in particular, is the reason for not having explicitly, in the r.h.s. of (19), terms corresponding to inverse processes, in which a parton with $x$ momentum fraction ends up in others with smaller momenta.

An alternative representation of (19) has been developed in [11], where their microscopical interpretation is more clear. According to this, instead of using direct $1 /(1-z)_{+}$regularization, it is shown how the virtual diagrams, responsible for parton wave function renormalization, are equivalent to real diagrams with negative sign. Using in fact Mueller cut-vertices technique [20], in addition to the ordinary real diagrams, leading to positive contribution to parton distributions variation, a negative term arises, corresponding to virtual gluon emission diagrams with exactly the same form for the unregularized parton splitting function as that of the real ones.

By helicity conservation at the quark-gluon vertex, and assuming $n_{f}$ different flavours for quarks $\left(j=1, \ldots, n_{f}\right)$ with two helicity states $(\lambda=+,-)$, the evolution equations for polarized quark distribution functions can be cast in the following form

$$
\begin{aligned}
\frac{d}{d \tau} q_{j \lambda}(x, \tau) & =\int_{x}^{1} \frac{d z}{z}\left\{\gamma_{q q}(z) q_{j \lambda}\left(\frac{x}{z}, \tau\right)+\frac{1}{2} \gamma_{q g}(z) G\left(\frac{x}{z}, \tau\right)\right\} \\
& -q_{j \lambda}(x, \tau) \int_{0}^{1} d z \gamma_{q q}(z) .
\end{aligned}
$$

Note that, for simplicity, we have assumed $G_{+}=G_{-}=G / 2$. We will come back on this point in the following. The equations for antiquarks are easily obtained by the previous one by substituting $q_{j \lambda} \leftrightarrow \bar{q}_{j \lambda}$. Similarly for the gluon unpolarized distribution $G(x, \tau)$ 
one has

$$
\begin{aligned}
\frac{d}{d \tau} G(x, \tau) & =\int_{x}^{1} \frac{d z}{z}\left\{\gamma_{g g}(z) G\left(\frac{x}{z}, \tau\right)+\sum_{j=1}^{n_{f}} \sum_{\lambda=+,-} \gamma_{g q}(z)\left[q_{j \lambda}\left(\frac{x}{z}, \tau\right)+\bar{q}_{j \lambda}\left(\frac{x}{z}, \tau\right)\right]\right\} \\
& -\frac{1}{2} G(x, \tau) \int_{0}^{1} d z\left[\gamma_{g g}(z)+2 n_{f} \gamma_{q g}(z)\right] .
\end{aligned}
$$

In the previous equations the splitting functions $\gamma_{A B}$ are defined by

$$
\begin{aligned}
\gamma_{q q} & =\frac{4}{3} \frac{1+z^{2}}{1-z}, \\
\gamma_{q g} & =\frac{1}{2}\left[z^{2}+(1-z)^{2}\right], \\
\gamma_{g q} & =\frac{4}{3} \frac{1+(1-z)^{2}}{z}, \\
\gamma_{g g} & =6\left[z(1-z)+\frac{1-z}{z}+\frac{z}{1-z}\right] .
\end{aligned}
$$

Note that all the divergences due to the singular behaviour of $\gamma_{A B}$ are explicitly cancelled once the terms with opposite sign, occurring in (24) and (25), are taken into account.

As already mentioned, AP equations in the form (24) (25) have a very clear and intuitive physical interpretation. Let us consider, for example, the $Q^{2}$ variation of a quark distribution function with momentum fraction $x, q_{j \lambda}(x)$ : from (24) we see that this is due to two terms, with opposite sign. The first one corresponds to the production of quarks with momentum $x$ from partons (gluons or quarks) with higher momentum fraction $y \geq x$, so it contributes with a positive sign. On the contrary, the second term accounts for the depletion of $x$ fraction quarks due to their decay in a quark with smaller momentum fraction $z x$ plus a gluon. In particular, notice that the same probability densities $\gamma_{A B}$ appear in both contributions, showing the physical soundness of the picture. Similar is the interpretation of (25).

As remarked in [11] this formulation also allows for an intriguing formal interpretation of $\mathrm{AP}$ equations as a set of transport Boltzmann equations if one regards the variable $\tau$ as the analogous of time variable. From this point of view the evolution in $Q^{2}$ of parton densities appear to be strictly equivalent to the evolution in time of statistical distributions corresponding to interacting particles, forming a system approaching equilibrium. To better illustrate this analogy it is useful to briefly review the Boltzmann transport equation formalism. 


\section{Statistical effects on parton distribution scaling behaviour}

As well-known, the Boltzmann set of equations describes the evolution to equilibrium states of systems composed by many particles of several species ( $i$ specie-index $i=$ $1, . ., n)$ mutually interacting [21]. Assuming for simplicity particles homogeneously and isotropically distributed, we can define the numerical distribution functions as

$$
n_{i}(\epsilon, t) \equiv g_{i}(\epsilon) f_{i}(\epsilon, t) \quad,
$$

with $\epsilon$ denoting the energy, $f_{i}(\epsilon, t)$ the statistical functions (they recover the usual Bose/Einstein or Fermi/Dirac at the thermal equilibrium), and $g_{i}(\epsilon)$ the level-densities (weights) corresponding to $\epsilon$. These last quantities should be fixed from the beginning, by studying the hamiltonian of the system. From (30) follows the expression for the total number-density of i-particles

$$
N_{i}(t)=\int \frac{d^{3} \vec{p}}{(2 \pi)^{3}} g_{i}(\epsilon) f_{i}(\epsilon, t)
$$

where $\vec{p}$ is the 3 -momentum, with $\vec{p}^{2}=\epsilon^{2}-m^{2}$. By using Eq. (30), the Boltzmann equations can be cast in the following form

$$
\mathcal{L} n_{i}=C_{i}[\mathbf{f}, \mathbf{g}]=C_{i}^{+}[\mathbf{f}, \mathbf{g}]-C_{i}^{-}[\mathbf{f}, \mathbf{g}] \quad i=1, \ldots, n,
$$

where $\mathbf{f} \equiv\left(f_{1}, \ldots, f_{n}\right), \mathbf{g} \equiv\left(g_{1}, \ldots, g_{n}\right), \mathcal{L}$ is the Liouville operator, and $C_{i}[\mathbf{f}, \mathbf{g}]$ is the so called collisional integral for the i-th particle specie. The latter is given by a thermal average of all possible processes which change the density of the i-th specie. Notice that in Eq. (32) we have defined $C_{i}^{+}[\mathbf{f}, \mathbf{g}]$ and $C_{i}^{-}[\mathbf{f}, \mathbf{g}]$ as the contributions corresponding to the interaction processes which create or destroy the i-th particle specie respectively. For simple three body processes $A \rightarrow B+C, B \rightarrow A+C$, if we are interested in describing, for example, the modification of $B$ population, the corresponding terms in $C_{B}[\mathbf{f}, \mathbf{g}]$ are the following

$$
\begin{aligned}
C_{B}^{+}[\mathbf{f}, \mathbf{g}] & -C_{B}^{-}[\mathbf{f}, \mathbf{g}]=\iint \frac{d^{3} \vec{p}_{A}}{2 \epsilon_{A}} \frac{d^{3} \vec{p}_{C}}{2 \epsilon_{C}}\left\{|\mathcal{M}(A \rightarrow B+C)|^{2} \frac{\delta\left(\epsilon_{A}-\epsilon_{B}-\epsilon_{C}\right)}{(2 \pi)^{2}}\right. \\
& \left.\times \delta^{3}\left(\vec{p}_{A}-\vec{p}_{B}-\vec{p}_{C}\right) n_{A}\left(\epsilon_{A}, t\right) g_{B}\left(\epsilon_{B}\right)\left[1 \pm f_{B}\left(\epsilon_{B}, t\right)\right] g_{C}\left(\epsilon_{C}\right)\left[1 \pm f_{C}\left(\epsilon_{C}, t\right)\right]\right\} \\
& -\iint \frac{d^{3} \vec{p}_{A}}{2 \epsilon_{A}} \frac{d^{3} \vec{p}_{C}}{2 \epsilon_{C}}\left\{|\mathcal{M}(B \rightarrow A+C)|^{2} \frac{\delta\left(\epsilon_{B}-\epsilon_{A}-\epsilon_{C}\right)}{(2 \pi)^{2}} \delta^{3}\left(\vec{p}_{B}-\vec{p}_{A}-\vec{p}_{C}\right)\right. \\
& \left.\times \quad n_{B}\left(\epsilon_{B}, t\right) g_{A}\left(\epsilon_{A}\right)\left[1 \pm f_{A}\left(\epsilon_{A}, t\right)\right] g_{C}\left(\epsilon_{C}\right)\left[1 \pm f_{C}\left(\epsilon_{C}, t\right)\right]\right\}
\end{aligned}
$$


where $|\mathcal{M}|^{2}$ are the squared moduli of transition amplitudes and the sign in the final state factors is positive/negative depending on the bosonic/fermionic nature of particles. In the limit of very small $f_{i}$ one has $\left(1 \pm f_{i}\right) \sim 1$ and the collisional term for very dilute systems is recovered.

Coming back to the analogy between AP equations and Boltzmann equations outlined in the previous section, it is physically reasonable to imagine that the AP evolution equations have to be modified for sufficiently low $x$. In this regime the nucleons are filled with a large number of quark-antiquark pairs and gluons (the sea) and thus, to take into account in the correct way the presence of this large number of partons, the decay processes should be considered in presence of a surrounding plasma of both Fermi and Bose particles. Corrections induced by quantum statistical effects to the scaling behaviour dictated by standard AP equations are therefore generally present, and in particular we expect that:

a) Pauli blocking will suppress the production of quarks and antiquarks with fraction $x$ corresponding to filled levels;

b) the gluon emission probability through bremsstrählung processes, considered in the standard picture leading to AP equations, will be enhanced by the contribution of induced-emission in presence of a rather relevant number of gluons in the sea.

These effects would favour the production of gluon-quark pairs with larger values of $x$ for the quarks and a smaller one for the gluon. Moreover the gluon conversion processes in $q-\bar{q}$ pairs are expected to be reduced.

As shown in (32), (33) in non-equilibrium statistical mechanics all these effects are simply included by multiplying the amplitudes modulus squared of the relevant processes, appearing in the collisional integral, by the factors $1-f$ or $1+f$ for each Fermi or Bose particle in the final state, with $f$ denoting the particle distribution functions without any level-density factor. In equilibrium conditions these $f$ reach the standard stationary Fermi-Dirac or Bose-Einstein form, while in general they depend on time. Thus, it is reasonable to expect that similar factors should be introduced in the generalized AP equations. In other words, standard AP equations correspond to a set of Boltzmann equations for a dilute system of partons, where statistical effects can be neglected: for higher parton densities, if we assume that this analogy still holds], it

2 It seems to us that the microscopical and fundamental character of the interpretation of AP equations as transport equations supports this assumption. 
follows that these effects, which are present in transport equations, should be present in scaling equations as well.

In the same spirit of (12) and (13) [5], we will parametrize the quark, antiquark and gluon distributions as

$$
\begin{aligned}
q_{j \lambda}(x, \tau) & =g_{j \lambda}(x) f_{j}^{\lambda}(x, \tau) \\
\bar{q}_{j \lambda}(x, \tau) & =\bar{g}_{j \lambda}(x) \bar{f}_{j}^{\lambda}(x, \tau) \\
G(x, \tau) & =g_{G}(x) f_{G}(x, \tau)
\end{aligned}
$$

where $g_{j \lambda}(x), \bar{g}_{j \lambda}(x)$ and $g_{G}(x)$ are weight functions, whereas $f_{j}^{\lambda}(x, \tau), \bar{f}_{j}^{\lambda}(x, \tau)$ and $f_{G}(x, \tau)$ are purely statistical distributions, which depending on $\tau$ cannot be assumed in principle to have equilibrium form. The explicit form for $g$-functions, which contains the divergency at $x=0$, should be fitted from experimental data, as in [5], or deduced from theoretical expected behaviour, like, for example, Regge theory. We stress that the factorized form (34)-(36), in particular the hypothesis that the singular functions $g_{j \lambda}, \bar{g}_{j \lambda}$ and $g_{G}$ do not depend on $\tau$ is compatible with predictions of both Regge theory and $Q C D$ for the behaviour of parton distributions at the end-point $x=0$. As it is well-known, in this regime one has

$$
p_{A}\left(x, Q^{2}\right) \sim \xi_{A}\left(Q^{2}\right) x^{-\alpha_{A}}
$$

with $\alpha_{A}$ not depending on $Q^{2}$, at least for large $Q^{2}$ [22].

Within the factorized expression (34)-(36) the final state factors are written in the form $1-f_{j}^{\lambda}, 1-\bar{f}_{j}^{\lambda}$ and $1+f_{G}$ for quarks, antiquarks and gluons respectively.

We are now able to introduce a set of generalized scaling equations for quarks and gluons. Here we will consider for simplicity the case in which the gluons are supposed not to have a significant net polarization in the nucleons with respect to the one carried by quarks. We will assume, therefore $G_{+}(x, \tau)=G_{-}(x, \tau)=G(x, \tau) / 2$. It should be pointed out that this approximation is consistent with the results obtained in [5] and [6], where it is argued that Pauli principle plays the essential role to generate the polarization of the quark sea. This approximation is instead less satisfactory in the framework of the different interpretation of the violation of Ellis-Jaffe sum rule based on the axial-vector current anomaly [23]. This latter case, in fact, would require a very large gluon polarization, i.e. $\Delta G=G_{+}-G_{-} \sim 3 \div 4$. Notice however that, as shown in [5], gluons are expected to be more numerous than quarks, due to their Bose nature, so in any case one has $\Delta G / G<<\Delta q / q$, which supports our approximation.

By helicity conservation at the quark-gluon vertex, it is easily seen that generalized evolution equations for polarized quark distribution functions get the following form 
|12

$$
\begin{aligned}
\frac{d}{d \tau} q_{j \lambda}(x, \tau) & =\int_{x}^{1} \frac{d z}{z}\left\{\gamma_{q q}(z) q_{j \lambda}\left(\frac{x}{z}, \tau\right)\left[1-f_{j}^{\lambda}(x, \tau)\right]\left[1+f_{G}\left(x\left(\frac{1}{z}-1\right), \tau\right)\right]\right. \\
& \left.+\frac{1}{2} \gamma_{q g}(z) G\left(\frac{x}{z}, \tau\right)\left[1-f_{j}^{\lambda}(x, \tau)\right]\left[1-\bar{f}_{j}^{-\lambda}\left(x\left(\frac{1}{z}-1\right), \tau\right)\right]\right\} \\
& -q_{j \lambda}(x, \tau) \int_{0}^{1} d z \gamma_{q q}(z)\left[1-f_{j}^{\lambda}(x z, \tau)\right]\left[1+f_{G}(x(1-z), \tau)\right] .
\end{aligned}
$$

The equations for antiquarks are easily obtained by the previous one by substituting $q_{j \lambda} \leftrightarrow \bar{q}_{j \lambda}$ and $f_{j}^{\lambda} \leftrightarrow \bar{f}_{j}^{\lambda}$. Similarly for the gluon distribution $G(x, \tau)$ one has

$$
\begin{aligned}
\frac{d}{d \tau} G(x, \tau) & =\int_{x}^{1} \frac{d z}{z}\left\{\gamma_{g g}(z) G\left(\frac{x}{z}, \tau\right)\left[1+f_{G}(x, \tau)\right]\left[1+f_{G}\left(x\left(\frac{1}{z}-1\right), \tau\right)\right]\right. \\
& +\sum_{j=1}^{n_{f}} \sum_{\lambda=+,-} \gamma_{g q}(z)\left[1+f_{G}(x, \tau)\right]\left\{q_{j \lambda}\left(\frac{x}{z}, \tau\right)\left[1-f_{j}^{\lambda}\left(x\left(\frac{1}{z}-1\right), \tau\right)\right]\right. \\
& \left.\left.+\bar{q}_{j \lambda}\left(\frac{x}{z}, \tau\right)\left[1-\bar{f}_{j}^{\lambda}\left(x\left(\frac{1}{z}-1\right), \tau\right)\right]\right\}\right\} \\
& -\frac{1}{2} G(x, \tau) \int_{0}^{1} d z\left\{\gamma_{g g}(z)\left[1+f_{G}(x z, \tau)\right]\left[1+f_{G}(x(1-z), \tau)\right]\right. \\
& \left.+\sum_{j=1}^{n_{f}} \sum_{\lambda=+,-} \gamma_{g g}(z)\left\{\left[1-f_{j}^{\lambda}(x z, \tau)\right]\left[1-\bar{f}_{j}^{-\lambda}(x(1-z), \tau)\right]\right\}\right\}
\end{aligned}
$$

Note that also in this case, as in (24) and (25) the divergent contributions due to $\gamma_{A B}$ exactly cancel. These generalized equations predict also a different, more complicated, evolution for momenta. By taking Mellin transform of both sides of (38) and (39), in fact, one sees that the standard scaling behaviour should be corrected by terms quadratic and cubic in distribution functions, which are not simply products of momenta of quarks and gluon densities.

Finally, as for the standard AP equations, the scaling behaviour for unpolarized quark distributions can be obtained by simply considering the sum $q_{j}(x, \tau)=q_{j+}(x, \tau)+$ $q_{j-}(x, \tau)$ (the same holds for antiquarks). Notice, however, that since the introduction of final state statistical factors spoils the linearity of the equations, the evolution of $q_{j}(x, \tau)$ will depend on both the polarized distribution functions and not simply on their sum. 


\section{Conclusions and remarks}

As suggested by some experimental results [8], [9], the Fermi/Bose nature of partons could sensibly affect the observable quantities in deep inelastic scattering on nucleons. This idea, already successfully applied in [5] and [6], mainly motivates our paper, in which a set of generalized scaling-law equations for parton distributions which take into account quantum statistics effects are suggested.

It is quite natural to think that quantum statistics may modify the scaling behaviour of parton distribution functions for rather small $x$ and high $Q^{2}$. This regime is in fact characterized by a large number of partons, which partially overlap their wave-functions, thus to correctly treat it one has to think in terms of parton-plasma dynamics, and the expected modifications to the standard AP evolution equations should have both dynamical (different processes) and/or statistical nature (statistical correlation between the wave functions).

The Gribov-Lipatov-Ryskin equations [4] represents a successful attempt to describe this system. It focus the attention only on the dynamical aspect of the problem, considering new interactions among partons which introduce in tha scaling-law nonlinear terms of the parton distribution functions. These processes, which become relevant with the increasing of the density, differently from the one considered in the standard AP approach involve two or more partons in the initial state (annihilation processes). In this paper we have stressed a different but complementar aspect, trying to introduce only the modifications to the evolution equation which are of genuine quantum statistical origine. Hence, a complete description of this region in the $x-Q^{2}$ plane should take into account both the results.

At low $x$, but still at high $Q^{2}$ (perturbative QCD regime), the bremsstrählung processes, responsible at leading-log level for scaling breaking, are likely supposed to occur in presence of such an overdense gas of partons. In this case Pauli blocking and gluon stimulated emission play a relevant role in parton distributions dynamics and thus in their scaling-law. We have introduced both this statistical effects to obtain a generalized evolution law, starting from the observation that an intriguing analogy holds between AP equations, at leading-log, and a set of Boltzmann transport equations for a dilute gas of partons [11], where a simple function of the scale variable $Q^{2}$ in $\mathrm{AP}$ equations plays the role of time parameter. Extending this analogy also to the case of a dense system, which is the case for the $x-Q^{2}$ region under study, one is naturally led to a new set of evolution equations in which statistical factors $\left(1-f_{j \lambda}(x, \tau)\right)$ or $\left(1+f_{G}(x, \tau)\right)$ appear in the r.h.s. of the evolution equation (collisional integral) to take into account the final state of the emitted parton. This approach implicitely suggests to 
consider a parton distribution $q_{j \lambda}(x, \tau)$ as the product of a pure weight factor $g_{j \lambda}(x, \tau)$ connected to the level-density and independent of $Q^{2}$, like for example suggested at low $x$ by the Regge theory [22], times the statistical distributions $f_{j \lambda}(x, \tau)$. According to this analogy and by virtue of Boltzmann $H$ theorem, one would naturally expect that the normalized parton distributions $f_{j \lambda}(x, \tau), \bar{f}_{j \lambda}(x, \tau)$ and $f_{G}(x, \tau)$ should approach stationary Fermi and Bose expressions as $Q^{2}$ increases. Remarkably, these conclusions seem to agree with the phenomenological results obtained in [5] and suggest that the thermalization process is rapid enough to essentially reach the equilibrium conditions at $Q^{2}=4 \mathrm{GeV}^{2}$. This question, together with the new equation for momenta of distributions (no more linear and simple like in AP equations) will be the subject for further publications.

Finally, we want to stress the difference of this approach with respect to the way in which the occurrence of Pauli blocking effects are perturbatively studied in the literature [24]. The single, independent parton picture, which is at the basis of the improved parton model, is only possible for quite large $x$, where the low density parton fluid which fills the hadron, allows to neglect the statistical correlations between partons due to the overlapping of their wave functions. This is not the case when we move to the low $x$ regime, and thus in this region this treatment is not completely justified. Alternative approaches, even if heuristic, as the one presented here, have to be investigated, analyzing first of all their predictions.

\section{Acknowledgements}

We thank Prof. Franco Buccella for encouraging this work and for his valuable comments. 


\section{References}

[1] G. Wolf, Preprint DESY 94-022 (February 1994).

[2] G. Altarelli and G. Parisi, Nucl. Phys. B126 (1977), 298.

V. N. Gribov and L. N. Lipatov, Sov. Jour. Nucl. Phys. 15 (1972), 438, 675.

L. N. Lipatov, Sov. Jour. Nucl. Phys. 20 (1975), 94.

Y. L. Dokshitzer, Sov. Phys. JETP 46 (1977), 641.

[3] E.A. Kuraev, L.N. Lipatov and V.S. Fadin, Sov. Phys. JETP 45 (1977), 199.

Ya.Ya. Balitskii and L.N. Lipatov, Sov. J. Nucl. Phys. 28 (1978), 822.

[4] L.V. Gribov, E.M. Levin and M.G. Ryskin, Phys. Rep. 100 (1983), 1.

[5] C. Bourrely, F. Buccella, G. Miele, G. Migliore, J. Soffer and V. Tibullo, Zeit. Phys. C62 (1994), 431.

[6] F. Buccella and J. Soffer, Mod. Phys. Lett. A8 (1993), 225; Europh. Lett. 24 (1993) 165; Phys. Rev. D48 (1993), 5416.

[7] K. Gottfried, Phys. Rev. Lett. 18 (1967), 1174.

[8] M.Arneodo et al. (New Muon Coll.), Phys. Rev. D50 (1994), R1.

[9] J. Ashman, et al. (European Muon Coll.), Phys. Lett. B206 (1988), 364; Nucl. Phys. B328 (1989), 1.

[10] P.L. Anthony et al. (E142 Coll.), Phys. Rev. Lett. 71 (1993), 959.

[11] J.C. Collins and Jianwei Qiu, Phys. Rev. D39 (1989), 1398.

[12] G. Mangano, G. Miele and G. Migliore, Preprint FERMILAB-Pub-93/380-A (December 1993).

[13] G. Preparata, P. Ratcliffe and J.Soffer, Phys. Rev. Lett. 66 (1991), 687.

[14] R. D. Field and R. P. Feynman, Phys. Rev. D15 (1977), 259.

[15] M. Bourquin et al., Zeit. Phys. C21 (1983), 27.

P. Ratcliffe, Phys. Lett. B242 (1990), 271. 
[16] J. Ellis and R. L. Jaffe, Phys. Rev. D9 (1974), 1444.

[17] C. Foudas et al., Phys. Rev. Lett. 64 (1990), 1207.

S.R. Mishra et al., Phys. Rev. Lett. 68 (1992), 3499.

S.A. Rabinowitz et al., Phys. Rev. Lett. 70 (1993), 134.

[18] G. Balocchi et al., (UA6 Coll.) preprint CERN-PPE/93-129, to appear in Phys. Lett. B.

P. Oberson et al., talk at the $28^{\text {th }}$ Recontres de Moriond, Les Arcs, March 1993.

M. Werlen et al., talk at the International Europhysics Conference on HEP, Marseille, July 1993.

[19] M. Shaevitz et al., (CCFR Coll.) talk at the Recontres de Physique de la Valle d'Aoste, La Thuile, March 1993, preprint NEVIS R-1491.

[20] A.H. Mueller, Phys. Rev. D18 (1978), 3705.

S. Gupta and A.H. Mueller, Phys. Rev. D20 (1979), 118.

[21] See, for example: R. C. Tolman, The Principles of Statistical Mechanics (1938), Oxford University Press.

[22] F. J. Ynduráin, The Theory of Quark and Gluon Interactions (1993), Springer Verlag.

[23] A. V. Efremov and O. V. Teryaev, Preprint JINR-EL-88-287.

G. Altarelli and G. G. Ross, Phys. Lett. B212 (1988), 391.

R. Carlitz, J. C. Collins and A. H. Mueller, Phys. Lett. 214 (1988), 229.

[24] D.A. Ross and C. T. Sachrajda, Nucl. Phys. B149 (1979), 497. 


\section{Figure Captions}

Fig. 1. The difference $F_{2}^{p}(x)-F_{2}^{n}(x)$ at $Q^{2}=4 G e V^{2}$ versus $x$. The experimental data are taken from [8] and the solid line represents the fit [5].

Fig. 2. The ratio $F_{2}^{n}(x) / F_{2}^{p}(x)$ at $Q^{2}=4 G e V^{2}$ versus $x$. The experimental data are taken from [8] and the solid line represents the fit [5].

Fig. 3. $x g_{1}^{p}(x)$ versus $x$. Data are from [9] and solid line from [5].

Fig. 4. $x g_{1}^{n}(x)$ versus $x$. Data are from [10] and solid line from [5]. 
Fig. 1

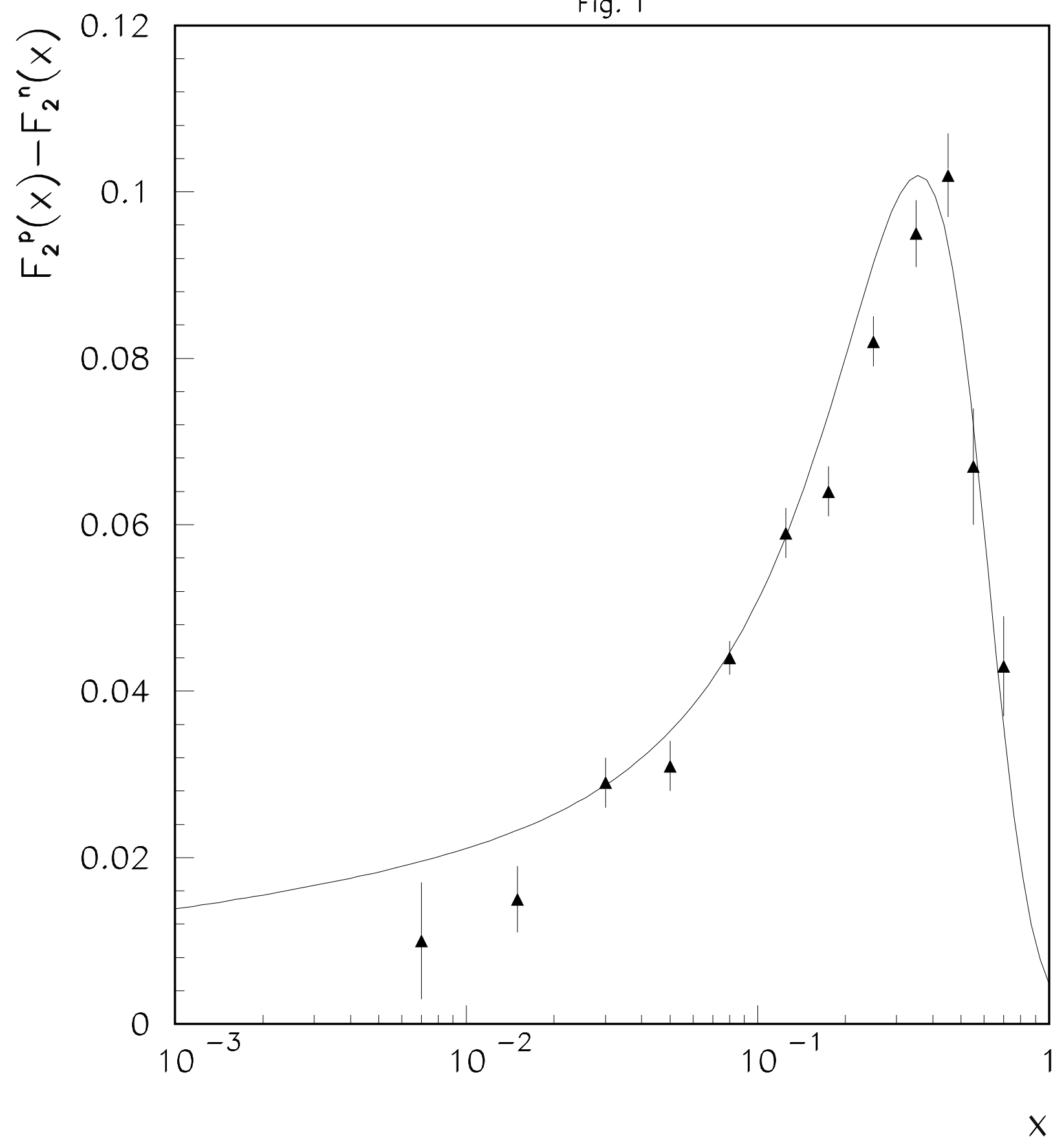


Fig. 2

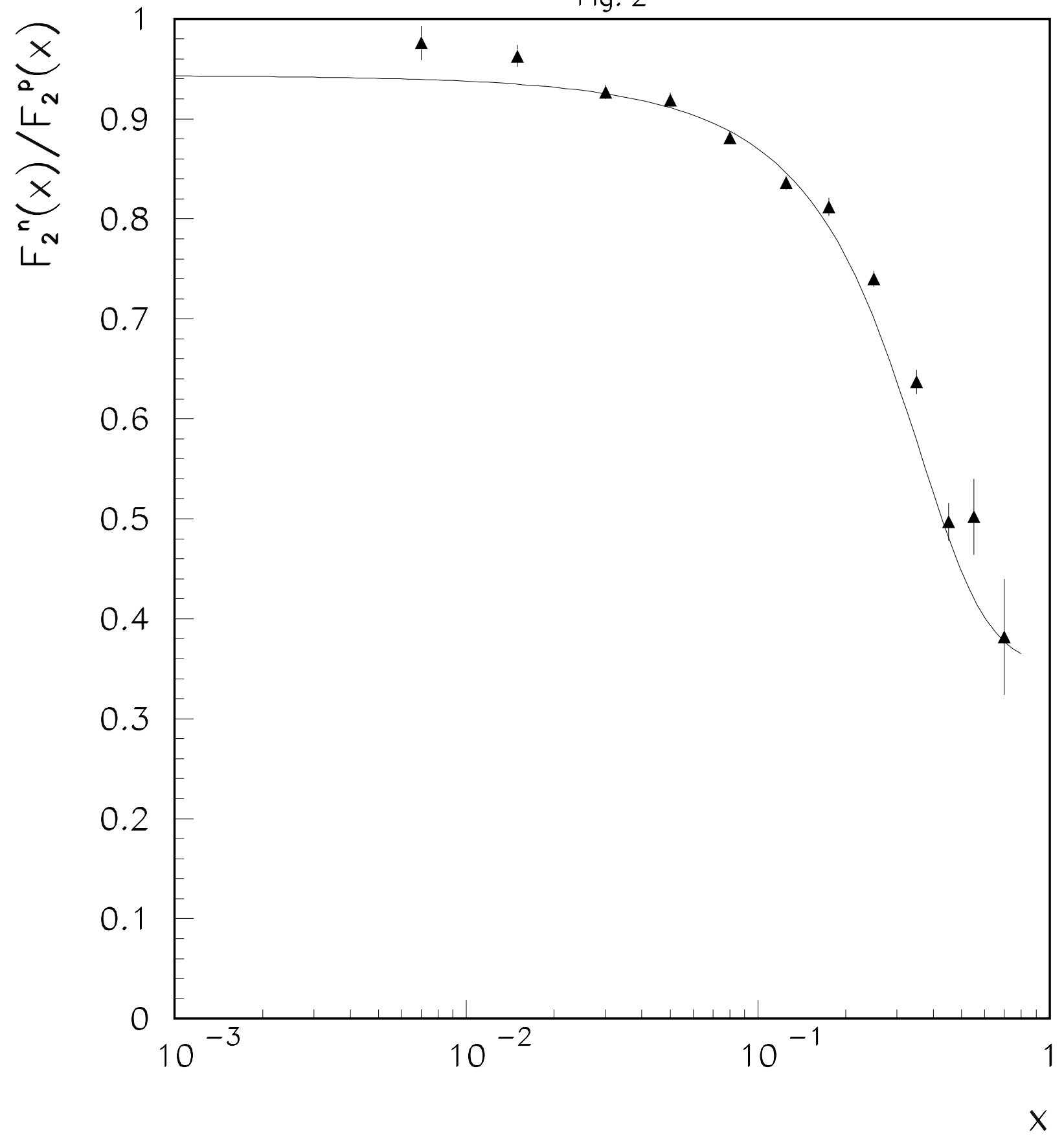


Fig. 3

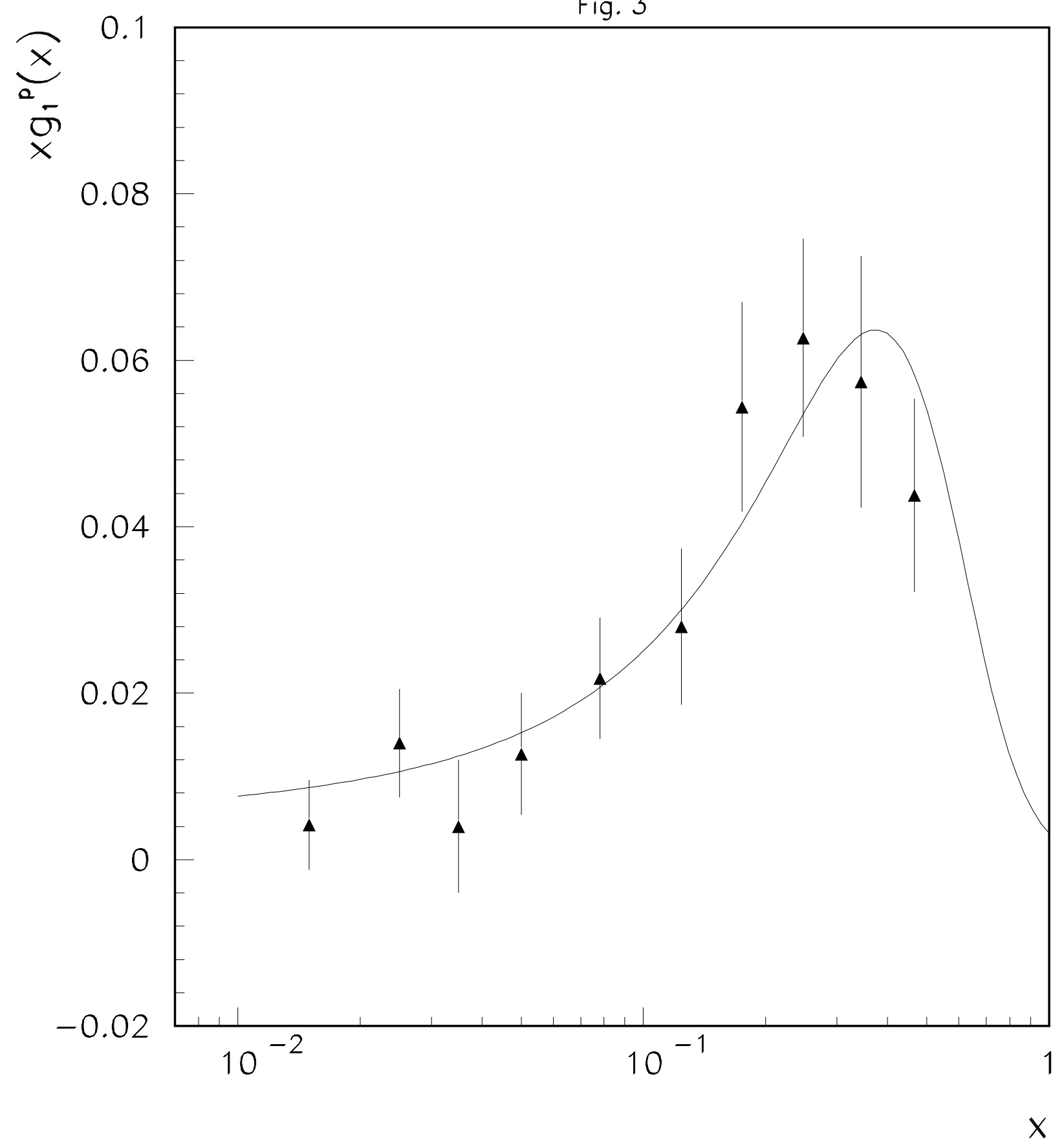


Fig. 4

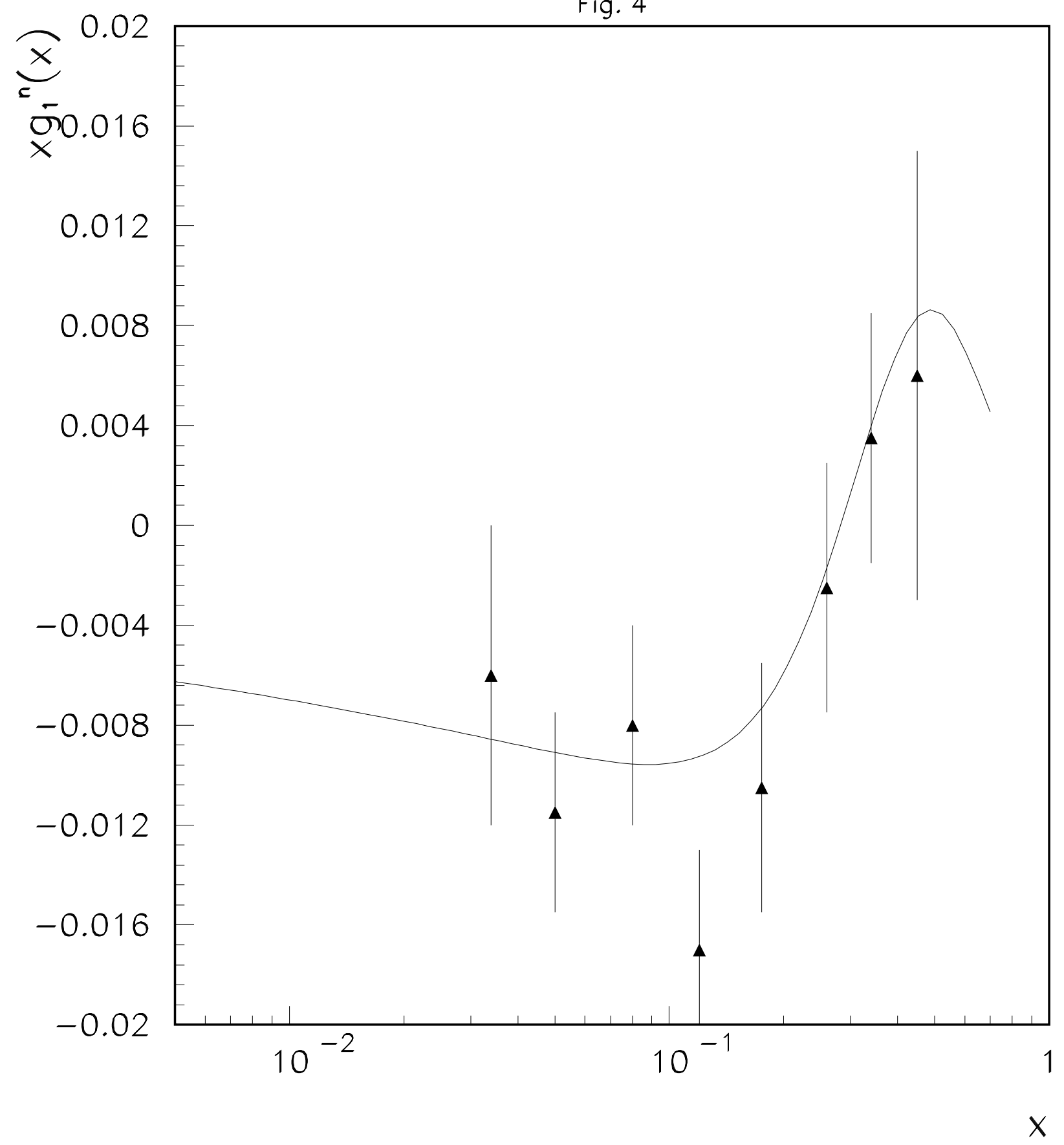

